\title{
Supplementation with long chain polyunsaturated fatty acids in treatment of atopic dermatitis in children
}

\author{
Maciej Kaczmarski ${ }^{1}$, Beata Cudowska², Małgorzata Sawicka-Żukowska ${ }^{3}$, Anna Bobrus-Chociej ${ }^{1}$ \\ 1Department of Pediatrics, Gastroenterology and Allergology, Medical University of Bialystok, Poland \\ Head: Prof. Maciej Kaczmarski MD, PhD \\ 2University Children's Teaching Hospital, Bialystok, Poland \\ Head: Janusz Pomaski MD, PhD \\ 3Department of Pediatric Oncology and Hematology, Medical University of Bialystok, Poland \\ Head: Prof. Maryna Krawczuk-Rybak MD, PhD
}

Postep Derm Alergol 2013; XXX, 2: 103-107

DOI: $10.5114 /$ pdia.2013.34160

\begin{abstract}
Some recent studies indicate that unsaturated fatty acids, components of cellular membranes and precursors of immunomodulators, play a significant role in the pathogenesis of some symptoms of atopic dermatitis. Since they cannot be synthesized by the human body, they must be provided with nutrition as the so called exogenous fatty acids: linoleic (a precursor of arachidonic acid) and $\alpha$-linolenic acid (a precursor of eicosapentaenoic acid (EPA) and docosahexaenoic acid (DHA)). Their deficiency facilitates the development of some disorders, e.g. of the cardiovascular system or of the nervous system, or becomes the cause of intensification of ailments in their course e.g. pruritus and dryness in atopic dermatitis. Though clinical examinations to date confirm the efficacy of fatty acid supplementation in treatment of atopic dermatitis, their results are not explicit.
\end{abstract}

Key words: atopic dermatitis, polyunsaturated fatty acids.

\section{Introduction}

The significance of lipids as a natural source of energy, lipid-soluble vitamines and fatty acids, being structural material synthesizing numerous biologically active compounds vital for the human organism, has been the subject of research for many years. Fatty acids are found in vegetable and animal fats and provided with food as, the so called exogenous fatty acids: linoleic (a precursor of arachidonic acid (AA)) and $\alpha$-linolenic acid (a precursor of eicosapentaenoic acid (EPA) and docosahexaenoic acid (DHA)) [1]. $\Omega$-3 fatty acids found in oil and fish meat and $\omega-6$ fatty acids obtained from seeds play a significant role in the organism. They maintain an appropriate structure, elasticity and functioning of cellular membranes as well as are essential in the synthesis of intracellular lipids in the corneous layer of epidermis. Additionally, they are also precursors of eicosanoids and have regulative properties [2]. The supply of these acids in a diet, especially the ratio of saturated acids to monounsaturated and polyunsaturated acids, decides about appropriate functioning of the human body [3]. The examinations prove that deficiency of unsaturated fatty acids facilitates the development of some disorders, e.g. of the cardiovascular system or cause the intensification of ailments in their course, e.g. in atopic dermatitis (AD) [2-6].

The results of clinical studies indicate that skin dryness, a basic therapeutic problem in AD, is caused mainly by the damage to the protective barrier of the epidermis, which consists of the aqueous and the lipid coat of dermis, a natural moisturizing factor and intracellular substance of the corneous layer of epidermis [2, 7]. This ailement frequently results from the insufficient supply of fatty acids in a diet. The clinical examinations carried out so far reveal that supplementation with preparations of fatty acids gives an opportunity to alleviate the symptoms of the disease and decrease the frequency of intensification of skin lesions in the course of $A D[2,5,8]$.

\section{Atopic dermatitis as a chronic inflammatory disease}

Atopic dermatitis is a chronic inflammatory skin disease associated, among others, with oversensitivity to

Address for correspondence: Beata Cudowska MD, PhD, University Children's Teaching Hospital, 17 Waszyngtona St, 15-274 Bialystok, Poland, phone: +48 857450 710, e-mail: becud@wp.pl Received: 3.09.2012, accepted: 28.02.2013. 
various allergens and environmental agents. The first skin lesions usually appear in the early childhood; however, they can persist and/or appear also in the adult life. Recently the prevalence of the disease has tended to increase and it occurs in about $10-20 \%$ of children and in $1-3 \%$ of adults. In many cases, AD lesions appearing in childhood can be the first step of 'an allergic march' and prognose allergic rhinitis and bronchial asthma, developing later in the adulthood [9-11].

A clinical manifestation of skin lesions is conditioned by genetic, environmental and immunological factors. Numerous examinations in vitro and in vivo confirm complex etiology of this disease, in which development and complicated immune mechanisms, including numerous cells and cytokines, are involved. Lymphocytes Th2, interleukins 4, 5, 13 (IL-4, IL-5, IL-13) and interferon- $\gamma$ (IFN- $\gamma$ ) play a special role in the development of this disease. The infiltrate of activated memory cells T CD4+ was proved in the analyses of skin bioptates with acute AD lesions, manifesting themselves as lichenization, reddening, pruritus, excoriations and exudates. Significantly more cells with the expression of mRNA IL-4, 5 and 13 were proved in the skin of patients with intensified lesions of the AD type in comparison with the skin of healthy subjects. In the skin subjected to long-term inflammation, remodeling takes place, resulting in lichenization, dryness of the marked intensity and the development of fibrous papules. Langerhans cells predominate in the skin infiltrate, though eosinophiles and lymphocytes $T$ are also present. Markedly more cells, expressing mRNA IL-5 and IFN- $\gamma$, were found at a low expression of IL-4 and IL-13. In $80 \%$ of cases, immune mechanisms of the disease were connected with the IgE-dependent reaction; in about 20\% - the IgE-independent reaction $[9,12,13]$.

The opinion that skin lesions resulted mainly from functioning disorders of the immune system and the change in the balance between lymphocyte populations Th2 and Th1/ThO has predominated for many years. It was regarded that this caused the development of the IgEdependent oversensitivity to airborne and food allergens (type I allergic reaction) in the acute phase of the disease. These phenomena were supposed to be responsible for the development of inflammatory changes within the dermis and epidermis (the so-called 'inside' theory). In recent years, studies have shown a genetically-conditioned dysfunction of the epidermal barrier associated with abnormal synthesis of structural proteins, high concentration of proteases and low activity of their inhibitors. Disorders of the epidermal barrier function, enabling allergens and irritating environmental agents to penetrate, are regarded as the primary factor in the etiopathogenesis of atopic eczema (the 'outside' theory) [7].

Lately, functional disorders of filaggrin, one of the key proteins conditioning a normal barrier function of the epidermis, have become the focus of researchers' attention. This function can be disturbed due to the mutation in the gene encoding filaggrin. The most frequently described mutations are R501X and 2282del4, predisposing, especially, to eczema lesions of an early onset as well as the severe and chronic course in patients with co-existing atopy [14].

Normal functioning of the epidermal barrier and, most of all, its hydration is associated with the metabolism of fatty acids and the appropriate supply of linolenic acid in a diet $[2,12]$.

\section{The role of long-chain polyunsaturated fatty acids (LCPUFAs) and metabolites of arachidonic acid in the pathogenesis of atopic dermatitis}

Recent studies indicate that fatty acids, components of cellular membranes and precursors of immunomodulators, play a significant role in the pathogenesis of atopic dermatitis. They are built into e.g. ceramides and metabolized to various metabolites in the dermis due to lypoand cyclooxygenase. A detailed mechanism of their activity has not been explained so far since the majority of studies refer to adults and some findings are contradictive [12].

$\Omega-3$ and $\omega-6$ acids, whose names come from the location of double binds counting from the end of the carbon chain, can be distinguished out of LC-PUFA. The synthesis of long-chain polyunsaturated fatty acids is conducted via two pathways. The former initiates the metabolism of linoleic acid (LA, 18:2 n-6) and leads to the formation of arachidonic acid (AA), and next prostaglandins, leukotrienes and thromboxanes; the latter $-\alpha$-linolenic acid (ALA, 18:3 n-3), leading to the synthesis of eicosapentaenoic acid (EPA, 20:5 n-3) and docosahexaenoic acid (DHA 22:6 n-3). The same enzymes, desaturases ( $\Delta-5$ and $\Delta$-6) and elongases, whose accurate functioning is of basic importance in the metabolism of LCPUFA, take part in both $L A$ and $A A$ transformations. The final products of the line n-6 metabolism have the proinflammatory, iatrogenic activity and they enhance cell proliferation. Metabolites of the line n-3 oppose these unfavorable activities, decreasing the intensity of an inflammatory process and platelet aggregation, protecting the endothelium and blood vessels, reducing the level of antioxidative stress, insulin sensitivity and improving a lipid profile. The EPA is regarded as the main anti-inflammatory factor due to its competitive activity with AA for metabolites. However, it has also been proved that DHA shows a potent antiinflammatory and immunomodulating activity [8, 12, 15].

In 1929, in the experimental animal studies, Burr et al. proved the relation between skin lesions, characterized by excessive dryness, reddening and the presence of abnormal keranocytes, and a deficit of LCPUFAs in a diet. The above abnormalities initially found in animals were also observed in humans. Children on cow's milk preparations, poor in fatty acids, demonstrated inflammatory skin lesions. Deficiency in LA caused dryness, red- 
dening and pruritus, leading to skin disorders. The increased index of endothelial cell proliferation, enhanced metabolism with an increase in the synthesis of sterol esters and then the presence of abnormal keratynocytes were found in histopathological examinations of skin biopatates.

In 1937, Brown and Hansen proved the decreased concentration of AA in the serum of children with atopic dermatitis, whereas the abnormal fatty acids profile and the decreased activity of $\Delta$-6-desaturase, an enzyme taking part in denaturation of LA to $\gamma$-linolenic acid (GLA), have been recently found in these patients. The GLA is a precursor of proinflammatory and immunomodulating agents, such as leukotrienes and prostaglandin E1, which is involved in maturation and differentiation of lymphocytes T, thus influencing the IgE production $[8,12]$.

A moderately increased concentration of $L A$ and a significantly decreased concentration of AA and DHA were reported in a Swedish study carried out in children with AD. Additionally, a positive correlation was proved between the concentration of immunoglobulin $E$ in the umbilical blood and the concentration of LA. The hypothesis was put forward that an increased risk of AD development could be caused by disorders of LA metabolism [12].

In other studies carried out as early as in the middle of the last century by Hansen et al., the decreased concentration of LA metabolites with the increased concentration of the acid in the blood serum was found in mothers breastfeeding infants with $A D$, compared to healthy infants' mothers [16]. The above findings were also confirmed by contemporary studies $[17,18]$. Their analysis shows that the decreased conversion of LA to its metabolites and the likely decreased building-in of fatty acids to phospholipids are observed in patients with AD. These abnormalities cause a decrease in the synthesis of prostaglandin E1 (PGE1), resulting in a low concentration of CAMP, which in consequence leads to the activation of specific structures of the immune system $[17,18]$.

None of the above studies enabled to answer the question whether the changes in fatty acids metabolism were the cause or consequence of atopic dermatitis. Published in 1994, Galli et al.'s studies determined the metabolites of fatty acids metabolism in the umbilical blood of children from the families with a positive history of atopic diseases and proved that changes in the metabolism of fatty acids caused no skin lesions [12, 19].

In the last ten years, much attention was paid to the studies of the genetic background of differences in the metabolism of LC-PUFA and disorders of the $\Delta-6$ desaturase activity [7]. To date, evaluating the fatty tissue, breast milk, umbilical blood and lymphocytes of the umbilical blood, fibroblasts and erythrocytes, the abnormal metabolism of fatty acids, involving a decreased concentration of LA metabolites, especially, GLA and AA at a normal concentration of LA, were proved in patients with $\operatorname{AD}[8,20]$.

\section{Significance of supplementation with preparations of $\gamma$-linolenic acid in the treatment of atopic dermatitis}

Long-chain polyunsaturated fatty acids are indispensible components of a diet because they cannot be synthesized by the human organism. The source of $n-3$ acids in a diet is mainly sea fish (herring, salmon, trout and others), sea food and oils (linen, rape-seed) and walnuts, whereas the main source of n-6 acids is corn, arachidic, cotton, soya and sesame oils, olive oil and plant seeds. Even in the case of a sufficient supply of linoleic and LA, this supplementation may be insufficient to synthesize the appropriate amount of LCPUFAs. This synthesis depends on the age, accompanying genetic and environmental conditions, etc. Poland is the country where deficiency of polyunsaturated fatty acids in a diet is especially high. Fish consumption in the adult population is insufficient and constitutes about $50 \%$ of the recommended consumption, whereas an average Pole provides his/her organism with approximately $1 / 5$ of demand for necessary fatty acids [1, 2, 4, 21].

Numerous studies performed on animal models and in people prove a favorable influence of the diet rich in polyunsaturated fatty acids in many diseases (e.g. cardiovascular, neurological, psychiatric, endocrinological, ophthalmological, dermatological or allergic diseases) $[1,4,22]$. More and more examinations confirm a positive effect of long-term supplementation with LC-PUFA in infancy on the development of the central nervous system [21].

The influence of polyunsaturated fatty acids on the development of diseases with allergic etiology has been examined numerous times as early as in the prenatal period. The anti-allergic activity of LC-PUFA has been analysed mostly in patients with bronchial asthma and $A D[2,12$, $13,23]$. It has been proved that if a diet is abundant in GLA, an increase in its metabolites: PGE1 and 15-hydroxyeicozatrienoic acid with anti-inflammatory activity is found in the dermis.

Additionally, it has been found that supplementation with preparations of fatty acids during pregnancy leads to a decreased incidence and intensity of $A D$ in children [24-26]. A detailed mechanism of the anti-allergic activity of LC-PUFA acids in AD has not been explained completely. The first findings on a favorable influence of fatty acids on the animal and human skin with AD come from the 1930s [16]. For the next decades, various preparations containing LC-PUFA were used to treat this disease, especially, those containing compounds with the various content of LA and GLA, usually improving the skin condition $[23,27] . \Omega-3$ and $\omega-6$ fatty acids provide the appropriate structure, elasticity and functionality of cell membranes and are vital for the synthesis of intracellular lipids in the corneous layer in the epithelium.

In the 1980s, more and more findings on supplementation with GLA preparations in the form of primrose oil 
(EPO), which contains $72 \%$ of LA and $9 \%$ of GLA, were published [12]. So far, many data containing various clinical experiments have been issued with only one systematic review of the literature on this subject. This metaanalysis dated 1989, which contained both published and unpublished data, is criticized for lack of detailed criteria with regard to unpublished data. It referred to the randomized studies and oral supplementation with fatty acids and did not confirm a favorable effect of such management on the severity of AD. According to authors, the efficacy, observed in some groups of patients, e.g. in infants and patients with severe $A D$, did not prove the need for or abandonment of this treatment $[24,28]$. Similarly, a new meta-analysis, made in 2004, did not confirm a significant influence of GLA on the severity of $A D$ [27]. The exception was a positive influence of fish oil on skin peeling and dryness.

However, a significant advantage of this preparation was found in other clinical randomized examinations performed by Schalin-Karrila et al. The amount of steroids applied topically was an important indicator of efficacy. It was observed that children treated with placebo used three times more steroid ointments than those treated with EPO [29]. Positive results were also demonstrated in a study by Biagi et al., who additionally found that this favorable effect lasted for a long time [30].

Dunstan et al. evaluated the influence of oral supplementation with preparations of fish oils in pregnant women suffering from atopic dermatitis, on the content of IgA, CD14, some cytokines (IL-5, IL-6, IL-10, TNF- $\alpha$, IFN- $\gamma$ ) and fatty acids in the breast milk after delivery. A significantly higher concentration of EPA and DHA was revealed in the milk of women taking preparations of fatty acids during pregnancy in comparison with controls. Additionally, it was proved that the course of $A D$ in children of these mothers was milder [31].

Galli et al. suggest that the supplementation with GLA plays a significant role in the early stage of $A D$ development, therefore administration of preparations of these acids seems extremely purposeful in the early childhood. Steward et al. claim that EPO changes slowly and gradually the composition of lipids in the cell membranes to the appropriate level [2].

Published in 2012, randomized examinations investigating the effect of supplementation with $\omega$-3 acids in pregnant women on the incidence of allergy in children, did not confirm a favorable influence of such management on the value of the total IgE concentration in the study group. A less frequent incidence of AD and sensitivity to hen's egg albumin was observed among children of mothers using supplementation with $\omega-3$ acids during pregnancy [23]. In other clinical studies with the same intervention, a decrease in the concentration of $A A$ and an increase in the ratio of EPA to DHA were revealed in the blood serum of women using supplementation with preparations of $\omega-3$ acid [32].
According to the opinion of the Experts' Group, published in 2011, pregnant and breastfeeding women should be supplemented with min. $200 \mathrm{mg}$ of DHA daily, whereas children above the age of 3 are recommended to consume long-chain fatty acids $\omega-3$ at a dose of 150-200 mg in $24 \mathrm{~h}$ [5].

At present, in the market there are numerous preparations containing acids from the $\omega-3$ group, though not all of them contain the appropriate dosage of these acids, taking into consideration their marked deficiency in a diet [2]. Polyunsaturated fatty acids are safe supplements of a diet, because they induce occasional, transitional and insignificant clinical side effects, usually, of the dyspeptic character. The DHA, whose activity was confirmed in numerous studies, including populations of healthy and ill people at various ages, seems to be the safest. The EPA, via its competition with AA, has some limitations when administered in infants (impaired growth). During exclusive breast feeding, the first 6 months of life, an additional intake of DHA seems not to be advised, because breast milk provides sufficient supplementation. The supplementation of feeding should be initiated after this period, introducing an additional source of $\omega$-3 acids e.g. fish into the diet [2]. Treatment of children with AD is multidirected with an elimination of agalactic, hypoallergic diet, in which it is not advisable to consume sea fish, nuts a rich source of LC-PUFA acids as well as strong allergens. Thus, these patients are additionally at risk of an insufficient intake of these acids and their supplementation in a diet is especially significant.

\section{Conclusions}

Based on available meta-analyses, it can be assumed that efficacy of supplementation with EFA in the treatment of AD is not completely documented. A positive effect of using these preparations in some age groups e.g. in AD children up to 1 year old can be observed, however the studies published so far neither confirm nor deny this thesis. Thus, it is advisable to conduct clinical studies aimed at proving a different influence of various fatty acids on the concentration of proinflammatory cytokines in children with atopic dermatitis. The confirmation of the immunological modulation by polyunsaturated fatty acids in these patients could help to decide whether it is necessary to introduce the accessory therapy of LC-PUFA acids in $A D$ treatment. The improved condition of the skin, decreased incidence of AD lesions and alleviated ailments like pruritus, could improve the quality of life of these children and their parents as well as this would have favorable financial results.

\section{References}

1. Karłowicz-Bodalska K, Bodalski T. Long chain polyunsaturated fatty acids - biological properties and the role in treatment. Postępy Fitoterapii 2007; 1: 46-56. 
2. Nowicki R, Barańska-Rybak W. Shark liver oil as a additional treatment in atopic dermatitis. Pol Merkur Lekarski 2007; 23: 312-3.

3. Cicero AF, Reggi A, Parini A, Borghi C. Application of polyunsaturated fatty acids in internal medicine: beyond the established cardiovascular effects. Arch Med Sci 2012; 8: 784-93.

4. Jabłońska-Trypuć A, Czerpak R. The metabolism of polyunsaturated fatty acids and the role in prophylaxis and treatment of blood diseases. Przegl Kardiodiabetol 2009; 4: 55-63.

5. Statement on supplementation with docosahexaenoic and omega-3 acids in population of pregnant and breast-feeding women and infants and children up to three years. Postępy Neonatologii 2011; 1.

6. Suliborska J, Bogdański P, Duda G, et al. An assessment of dietary intake and state of nutritional in hypertensive patients from rural and urban areas of Greater Poland. Ann Agric Environ Med 2012; 19: 339-43.

7. Samochocki Z. Epidermal barrier dysfunction in development and course of atopic dermatitis. Alergia 2010; 4: 429.

8. Horrobin DF. Fatty acid metabolism in health and disease: the role of delta-6-desaturase. Am J Clin Nutr 1993; 57 (5 Suppl): 732S-6S.

9. Silny W, Czarnecka-Operacz M, Gliński W, et al. Atopic dermatitis - current views on pathomechanism, diagnostic and treatment. Statement of Polish Society of Dermatology. Postep Derm Alergol 2010; 28: 365-83.

10. Żukiewicz-Sobczak W, Krasowska E, Zwoliński J, et al. Allergic diseases - current state of knowledge. Postep Derm Alergol 2012; 29: 451-5.

11. Hallas TE, Gislason T, Gislason D. Mite allergy and mite exposure in Iceland. Ann Agric Environ Med 2011; 18: 13-7.

12. Horrobin DF. Essential fatty acid metabolism and its modification in atopic eczema. Am J Clin Nutr 2000; 71 (1 Suppl): 367S-72S.

13. Leung DJM, Boguniewicz M, Howell MD, et al. New insights into atopic dermatitis. J Clin Invest 2004; 113: 651-7.

14. Kurowski M, Kowalski ML. The role of filaggrin in pathomechanism of allergic diseases. Alergia Astma Immunologia 2009; 15: 95-100.

15. Ziboh VA, Craig CM, Cho Y. Metabolism of polyunsaturated fatty acids by skin epidermal enzymes; generation of antiinflammatory and antiproliferative metabolites. Am J Clin Nutr 2000; 71 (Suppl): 361S-6S.

16. Hansen AE, Knott EM, Wiese HF, et al. Eczema and essential fatty acids. Am J Dis Child 1947; 73: 1-18.

17. Thijs C, Muller A, Rist L, et al. Fatty acids in breast milk and development of atopic eczema and allergic sensitization in infancy. Allergy 2011; 66: 58-67.

18. Hoppu U, Rinne M, Lampi AM, et al. Breast milk fatty acid composition is associated with development of atopic dermatitis in the infant. J Pediatr Gastroenterol Nutr 2005; 41: 335-8.

19. Galli E, Picardo M, Chini L, et al. Analysis of polyunsaturated fatty acids in newborn sera: a screening tool for atopic disease? Br J Am Acad Dermatol 1994; 130: 752-6.

20. Van Gool CJAW, Zeegers MPA, Thijs C. Oral essential fatty acid supplementation in atopic dermatitis - a meta-analysis of placebo-controlled trials. Br I Dermatol 2004; 150: 728-40.

21. Jańczyk W, Socha P. Clinical effect of supplementation of polyunsaturated fatty omega-3 acids. Standardy Medyczne 2009; 4 (6).

22. Tehle ME, Hanczaruk M, Schwarz SC, et al. Effects of polyunsaturated fatty acids on isolated canine peripheral blood mononuclear cells and cytokine expression (IL-4, IFN-gamma, TGF-beta) in healthy and atopic dogs. Vet Dermatol 2010; 21: $112-7$.

23. Shield MJ, Wilson AM, Horrobin DF, et al. Essential fatty acid supplementation in atopic dermatitis. Lancet 1993; 342: 377-8.

24. Palmer DJ, Sullivan T, Gold MS, et al. Effect of n-3-long polyunsaturated fatty acid supplementation in pregnancy on infants' allergies in first year of life: randomized controlled trial. BMJ 2012; 344: e184.

25. Blumer N, Renz H. Consumption of [omega]3-fatty acids during perinatal life: role in immunomodulation and allergy prevention. J Perin Med 2007; 35 (Suppl 1): S12-8.

26. Wijga A, van Houwelingen AC, Smith HA, et al. Fatty acids in breast milk of allergic and non-allergic mothers. The PLAMA birth cohort study. Pediatr Allergy Immunol 2003; 14: 156-62.

27. van Gool CJAW, Thijs C, Henquet CJM, et al. Gamma-linolenic acid supplementation for prophylaxis of atopic dermatitis a randomized controlled trial in infants at high familial risk. Am J Clin Nutr 2003; 77: 943-51.

28. Morse PF, Horrobin DF, Manku MS, et al. Meta-analysis of placebo-controlled studies of the efficacy of Epogam in the treatment of atopic eczema. Relationship between plasma essential fatty acid changes and clinical response. Br J Dermatol 1989; 121: 75-90.

29. Schalin-Karrila M, Mattila L, Jansen CT, et al. Evening primrose oil in the treatment of atopic eczema: effect on clinical status, plasma phospholipid fatty acids and circulating blood prostaglandins. Br J Dermatol 1987; 117: 11-9.

30. Biagi PL, Bordoni A, Masi M, et al. A long-term study on the use of evening primrose oil (Efamol) in atopic children. Drugs Exp Clin Res 1988; 14: 285-90.

31. Dunstan JA, Mori TA, Barde A, et al. Fish oil supplementation in pregnancy modifies neonatal allergen-specific immune responses and clinical outcomes in infants at high risk of atopy: a randomized, controlled trial. J Allergy Clin Immunol 2003; 112: 1178-84.

32. Warstedt K, Furuhjelm C, Duchen K, et al. The effects of omega-3 fatty acid supplementation in pregnancy on maternal eicosanoid, cytokine, and chemokine secretion. Ped Res 2009; 66: 212-7. 